\title{
Assessing Blood Flow in an Intracranial Stent: A Feasibility Study of MR Angiography Using a Silent Scan after Stent- Assisted Coil Embolization for Anterior Circulation Aneurysms
}

\author{
(D) R. Irie, M. Suzuki, M. Yamamoto, N. Takano, Y. Suga, (D) M. Hori, K. Kamagata, M. Takayama, M. Yoshida, S. Sato, N. Hamasaki,
} H. Oishi, and S. Aoki

\begin{abstract}
BACKGROUND AND PURPOSE: Blood flow in an intracranial stent cannot be visualized with 3D time-of-flight MR angiography owing to magnetic susceptibility and radiofrequency shielding. As a novel follow-up tool after stent-assisted coil embolization, we applied MRA by using a Silent Scan algorithm that contains an ultrashort TE combined with an arterial spin-labeling technique (Silent MRA). The purpose of this study was to determine whether Silent MRA could visualize flow in an intracranial stent placed in the anterior circulation.
\end{abstract}

MATERIALS AND METHODS: Nine patients treated with stent-assisted coil embolization for anterior circulation aneurysms underwent MRAs (Silent MRA and TOF MRA) and x-ray digital subtraction angiography. MRAs were performed in the same session on a 3T unit. Two neuroradiologists independently reviewed the MRA images and subjectively scored flow in a stent as 1 (not visible) to 4 (excellent) by referring to the latest $\mathrm{x}$-ray digital subtraction angiography image as a criterion standard.

RESULTS: Both observers gave MRA higher scores than TOF MRA for flow in a stent in all cases. The mean score for Silent MRA was $3.44 \pm$ 0.53 , and for TOF MRA, it was $1.44 \pm 0.46(P<.001)$.

CONCLUSIONS: Silent MRA was able to visualize flow in an intracranial stent more effectively than TOF MRA. Silent MRA might be useful for follow-up imaging after stent-assisted coil embolization, though these study results may be only preliminary due to some limitations.

E ndovascular therapy for intracranial aneurysms has been widely used since the International Subarachnoid Aneurysm Trial. ${ }^{1}$ The number of cases of coil embolization for aneurysms is increasing, and the stent-protection technique has widened the applicability to cases that had been otherwise difficult to treat with conventional coil embolization. ${ }^{2}$ Nevertheless, there is a risk of coil compaction or in-stent restenosis after stent-assisted coil embolization. X-ray digital subtraction angiography is the optimal technique used to examine these adverse events, and it is commonly used as a follow-up tool after using an intracranial stent. However, DSA presents some unavoidable risks related to the catheter procedure, radiation, and contrast media. ${ }^{3-5}$

3D time-of-flight MR angiography is widely used for the assessment of cerebral vascular diseases and has also been examined

Received August 18, 2014; accepted after revision October 29.

From the Departments of Radiology (R.I., M.S., N.T., M.H., K.K., M.T., M. Yoshida, S.S., N.H., S.A.) and Neurosurgery (M. Yamamoto, Y.S., H.O.), Juntendo University Hospital, Tokyo, Japan; and Department of Neuroendovascular Therapy (H.O.), Juntendo University School of Medicine, Tokyo, Japan.

Please address correspondence to Ryusuke Irie, MD, Department of Radiology, Juntendo University Hospital, 3-1-3 Hongo, Bunkyo-ku, Tokyo 113-8431, Japan; e-mail: ririe@juntendo.ac.jp

- Indicates open access to non-subscribers at www.ajnr.org

http://dx.doi.org/10.3174/ajnr.A4199 as a noninvasive substitute for DSA. ${ }^{6-9}$ These studies generally reported difficulty in visualizing flow in a stent with TOF MRA because of magnetic susceptibility and radiofrequency shielding, though some beneficial aspects were observed in assessing the residual lumen of aneurysms. As a novel follow-up tool after stent-assisted coil embolization, we applied MRA by using a Silent Scan algorithm (GE Healthcare, Milwaukee, Wisconsin) that contains an ultrashort TE combined with an arterial spin-labeling technique (Silent MRA). In this situation, visualizing flow means visualizing arterial geometry and patency. It does not mean directly visualizing blood flow rate. The purpose of this study was to determine whether Silent MRA can visualize flow in an intracranial stent placed at the anterior circulation.

\section{MATERIALS AND METHODS}

Patients treated by stent-assisted coil embolization for unruptured aneurysms in the anterior circulation were examined, and 10 consecutive patients who underwent 2 types of MRAs (Silent MRA and TOF MRA) from March 1 to June 30, 2014, were included in this study. One patient with an anterior communicating artery aneurysm was excluded because the patient experienced stent occlusion during the follow-up period. Written informed consent was not required for this study because of its retrospective observational design. All 9 patients were treated with Enterprise VRD (Codman \& Shurtleff, Raynham, Massachusetts). Endovas- 


\begin{tabular}{|c|c|c|c|c|c|c|c|c|}
\hline $\begin{array}{l}\text { Case } \\
\text { No. }\end{array}$ & $\begin{array}{l}\text { Age } \\
\text { (yr) }\end{array}$ & Sex & Aneurysm Location & Stent Configuration $^{a}$ & $\begin{array}{l}\text { Interval between } \\
\text { DSA and MRA }\end{array}$ & $\begin{array}{c}\text { Aneurysm Occlusion } \\
\text { Status }\end{array}$ & $\begin{array}{c}\text { Scores of } \\
\text { Silent MRA }\end{array}$ & $\begin{array}{l}\text { Scores of } \\
\text { TOF MRA }^{b}\end{array}$ \\
\hline 1 & 65 & $\mathrm{~F}$ & Rt. ICA paraclinoid & Clinoid-terminal & $13 \mathrm{mo}$ & NR & $4 / 3$ & $1 / 1$ \\
\hline 2 & 75 & $M$ & Rt. ICA-AchoA & Supraclinoid-MCA & $15 \mathrm{mo}$ & $\mathrm{CO}$ & $4 / 3$ & $2 / 2$ \\
\hline 3 & 74 & $\mathrm{~F}$ & Rt. ICA paraclinoid & Cavernous-supraclinoid & $7 \mathrm{mo}$ & $\mathrm{CO}$ & $4 / 4$ & $1 / 1$ \\
\hline 4 & 59 & M & Lt. ICA bifurcation & Supraclinoid-MCA & 1 day & $\mathrm{CO}$ & $3 / 3$ & $2 / 1$ \\
\hline 5 & 45 & $\mathrm{~F}$ & Rt. ICA paraclinoid & Cavernous-terminal & $7 \mathrm{mo}$ & $\mathrm{CO}$ & $4 / 4$ & $2 / 2$ \\
\hline 6 & 50 & $\mathrm{~F}$ & Rt. ICA paraclinoid & Cavernous-terminal & 1 day & NR & $4 / 3$ & $1 / 1$ \\
\hline 7 & 51 & $\mathrm{~F}$ & Lt. ICA cavernous & Cavernous-clinoid & $12 \mathrm{mo}$ & $\mathrm{CO}$ & $2 / 3$ & $1 / 1$ \\
\hline 8 & 61 & $\mathrm{~F}$ & Rt. ICA-PcomA & Supraclinoid-terminal & 2 days & NR & $4 / 4$ & $2 / 2$ \\
\hline 9 & 64 & $M$ & Lt. ICA paraclinoid & Cavernous-supraclinoid & $12 \mathrm{mo}$ & NR & $3 / 3$ & $2 / 1$ \\
\hline
\end{tabular}

Note:-AchoA indicates anterior choroidal artery; PcomA, posterior communicating artery; Clinoid, clinoid segment of ICA; terminal, terminal segment of ICA; supraclinoid, supraclinoid segment of ICA; Cavernous, cavernous segment of ICA; NR, neck remnant; CO, complete occlusion; Rt., right; Lt., left.

a Proximal-distal marker of a stent.

${ }^{\mathrm{b}}$ Scores of observers $1 / 2$.

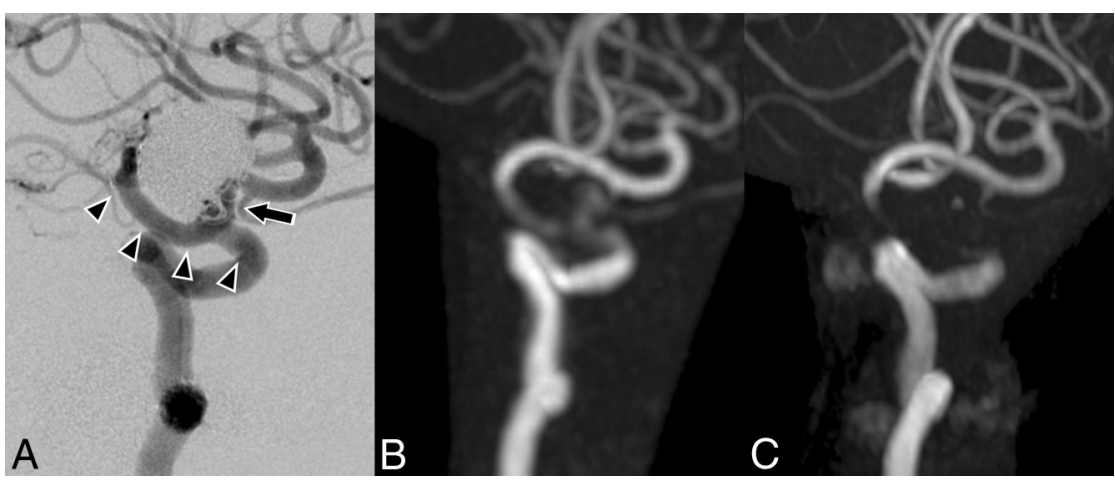

FIG 1. Case 1. A 65-year-old woman with a right paraclinoid internal carotid artery aneurysm was treated by stent-assisted coil embolization in March 2011. The latest DSA was performed in February $2013(A)$. Silent MRA (B) and TOF MRA (C) were performed in March 2014. The clinoidto-terminal segment of the internal carotid artery is covered by a stent (arrowheads). The anatomic outcome of the aneurysm in DSA is a neck remnant (arrow). Our subjective scores of Silent MRA are 4 and 3. The scores of TOF MRA are 1 and 1 . In this case, the Silent MRA depiction of the neck remnant is also better than that of TOF MRA.

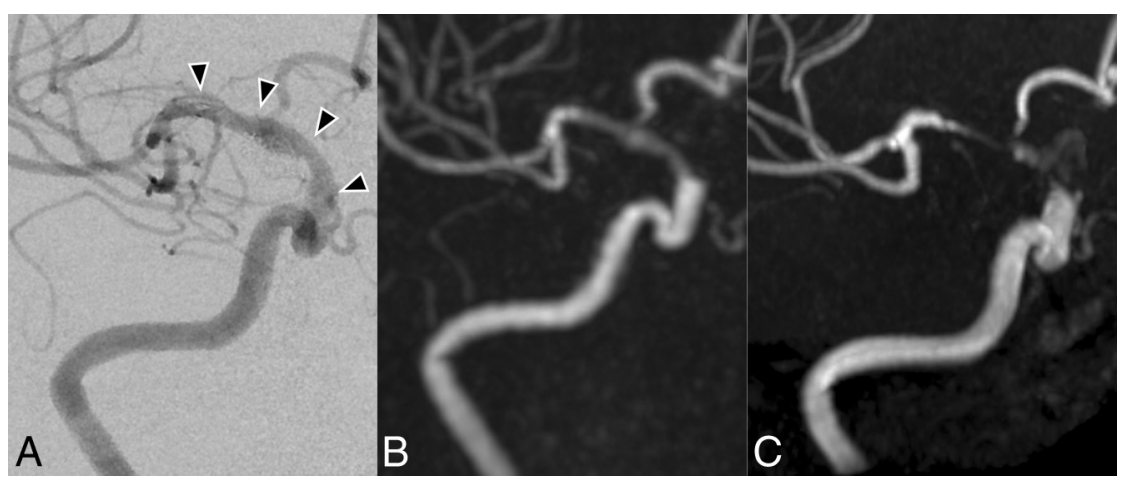

FIG 2. Case 2. A 75-year-old man with a right anterior choroidal artery aneurysm was treated in June 2010. The latest DSA was performed in December $2012(A)$. Silent MRA (B) and TOF MRA $(C)$ were performed in March 2014. The supraclinoid segment of the internal carotid artery to middle cerebral artery is covered by a stent (arrowheads). The anatomic outcome of the aneurysm in DSA is complete occlusion. Our subjective scores of Silent MRA are 4 and 3. The scores of TOF MRA are 2 and 2.

cular therapy and examinations were performed with the following angiographic systems: Axiom Artis dTA (Siemens, Erlangen, Germany) until February 2014 and Artis Q BA Twin (Siemens) from March 2014 to present. Anatomic outcomes of the aneurysm in the latest DSA were complete occlusion in 5 cases and neck remnant in 4 cases. Patient data are shown in the Table.

Both MRAs were performed in the same session on a 3T unit (Discovery MR750w; GE Healthcare). Silent MRA is only appli- cable to 3T MR imaging. The parameters for acquisition in the Silent MRA were as follows: TR/TE, 1116.4/0.016 ms; flip angle, $5^{\circ}$; FOV, $180 \times 180 \mathrm{~mm}$; matrix, $150 \times 150$; section thickness, $1.2 \mathrm{~mm}$; NEX, 1.5; bandwidth, $\pm 20 \mathrm{kHz}$; and acquisition time, 7 minutes 40 seconds. TOF MRA was performed with the following parameters: TR/TE, 19/2.9 ms; flip angle, $15^{\circ}$; FOV , $200 \times 200 \mathrm{~mm}$; matrix, $416 \times 192$; section thickness, $1.2 \mathrm{~mm}$; NEX, 1; bandwidth, $\pm 41.7 \mathrm{kHz}$; and acquisition time, 3 minutes 31 seconds. Although the details of the Silent Scan algorithm were undisclosed, the arterial spin-labeling technique is used as a preparation pulse, and data acquisition is based on a $3 \mathrm{D}$ radial scan. ${ }^{10}$ In the Silent Scan, a control image is first scanned before the labeling pulse, followed by a labeled image. The control and labeled images are subtracted to yield an angiographic image.

Two neuroradiologists independently reviewed the MRAs and rated the visual conditions around the flow in each stent subjectively on a 4-point scale as follows: 1 , not visible (almost no signal in the stent); 2, poor (structures are slightly visible but with significant blurring or artifacts, not diagnostic); 3, good (goodquality diagnostic information with minimal blurring or artifacts); or 4, excellent (excellent-quality diagnostic information; the shape of depiction is nearly equal to that of DSA). The scores of the 2 observers were averaged, and a paired $t$ test was performed in the statistical analysis of the subjective scores for flow in a stent. The latest DSA image was used as a reference standard. The average of the interval between the latest DSA and MRAs was 7.33 months (range, 1 day to 15 months), and the interval between the aneurysm treatment and MRAs was 18.67 months (range, 1 day to 45 months). The level of interobserver agreement in the evaluation was analyzed by weighted $\kappa$ statistics. 


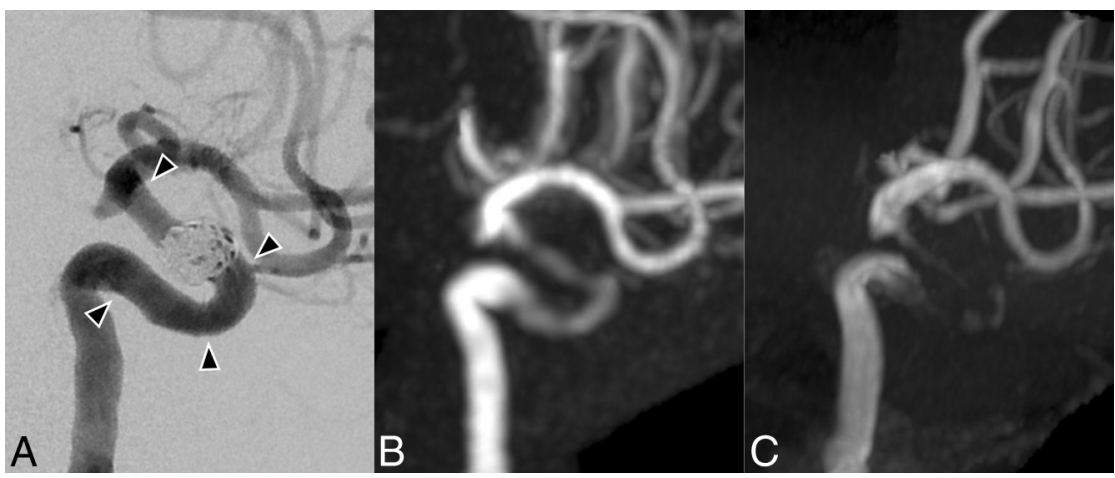

FIG 3. Case 3. A 74-year-old woman with a right paraclinoid internal carotid artery aneurysm was treated in October 2013. A DSA image $(A)$ was obtained right after the treatment. Silent MRA $(B)$ and TOF MRA (C) were performed in May 2014. The cavernous-to-supraclinoid segment of the internal carotid artery is covered by a stent (arrowheads). The anatomic outcome of the aneurysm in DSA is complete occlusion. Our subjective scores of Silent MRA are 4 and 4 . The scores of TOF MRA are 1 and 1 .
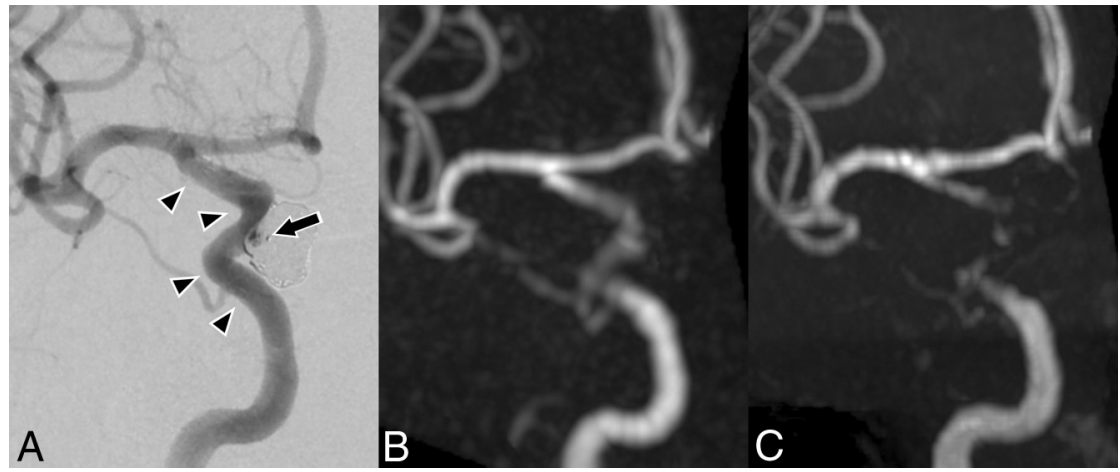

FIG 4. Case 6. A 50-year-old woman with a right paraclinoid internal carotid artery aneurysm was treated in June 2012. Silent MRA ( $B$ ) and TOF MRA $(C)$ were performed in June 2014, and DSA $(A)$ was performed the next day. The cavernous-to-terminal segment of the internal carotid artery is covered by a stent (arrowheads). The anatomic outcome of the aneurysm in DSA is a small neck remnant (arrow). Our subjective scores of Silent MRA are 4 and 3. The scores of TOF MRA are 1 and 1 .

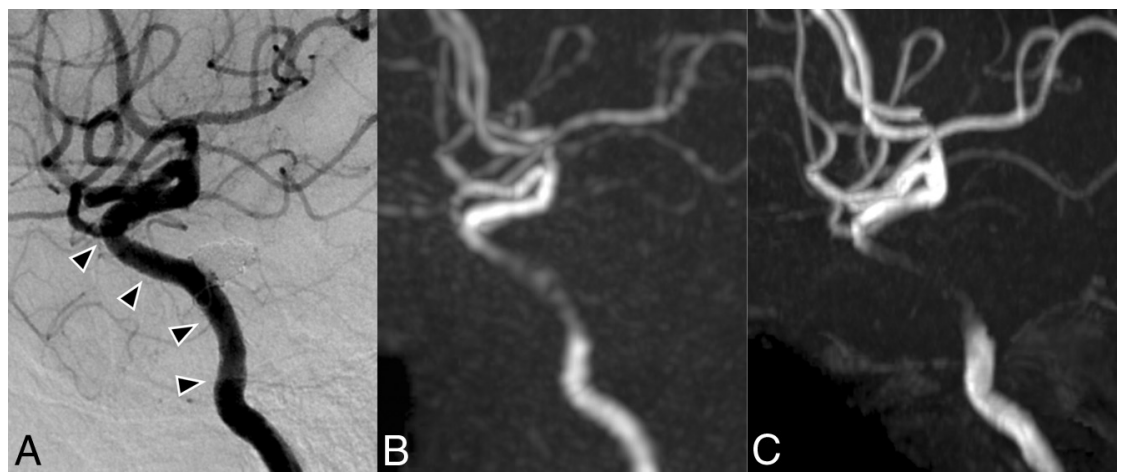

FIG 5. Case 7. A 51-year-old woman with a left cavernous internal carotid artery aneurysm was treated in May 2012. The latest DSA was performed in June $2013(A)$. Silent MRA (B) and TOF MRA (C) were performed in June 2014. The cavernous-to-clinoid segment of the internal carotid artery is covered by a stent (arrowheads). The anatomic outcome of the aneurysm in DSA is complete occlusion. In this case, the overall image quality of Silent MRA is not good, but visualization of the flow in a stent is still better than that of TOF MRA. Our subjective scores of Silent MRA are 2 and 3. The scores of TOF MRA are 1 and 1.

\section{RESULTS}

The mean score of Silent MRA was $3.44 \pm 0.53$, and for TOF MRA, it was $1.44 \pm 0.46(P<.001)$. Both observers gave Silent MRA higher scores than TOF MRA for flow in a stent in all cases. Interobserver agreement was $\operatorname{good}(\kappa=0.963)$. Of note, the Silent MRA depictions of the neck remnant in 4 cases were similar to those of DSA, while TOF MRA showed only a small depiction of the neck remnant. Figures 1-5 show the DSA, Silent MRA, and TOF MRA images for each case, respectively. Silent MRA and TOF MRA images were processed to maximum intensity projections, and surrounding vessels were removed to make them similar to those on DSA.

\section{DISCUSSION}

In this study, we found that Silent MRA was superior to TOF MRA for visualizing flow in a stent in all 9 observed patients with anterior circulation aneurysms.

In the anterior circulation, the tortuosity and diameter of the vessel are different from those in the posterior circulation. The effect of magnetic susceptibility by stent or coil may differ between the anterior and posterior circulations. Thus, we believe that there is a need to distinguish these regions when assessing flow in a stent.

The most important characteristic of a Silent Scan is the ultrashort TE (TE = $0.016 \mathrm{~ms}$ ). Ultrashort TE imaging can decrease magnetic susceptibility and visualize short T2 tissues such as the musculoskeletal system, lung parenchyma, or carotid plaque. ${ }^{11-13}$ In Silent MRA, the ultrashort TE can minimize the phase dispersion of the labeled blood flow signal in the voxel space and decrease magnetic susceptibility. Accordingly, the artifacts from stents or coils are diminished; this change enables visualization of flow in a stent. The effect of the disturbed flow in TOF MRA may also decrease. Gönner et $\mathrm{al}^{14,15}$ reported that ultrashort TE reduced the artifacts after coiling or stent placement, though a TE of $2.4 \mathrm{~ms}$ was used in that study.

In Silent MRA, the angiographic image is obtained by subtraction of images scanned before and after labeling. Thus, static tissue such as a thrombus cannot be detected in Silent MRA. Conversely, in TOF MRA, thrombus in a stent might be detected as a high-signal-intensity area. Compared with TOF MRA, Silent MRA images are easy to interpret because they consist of only the labeled blood flow signal. Takayama et $\mathrm{al}^{16}$ reported that contrast-enhanced MRA is 
useful when visualizing flow in a stent, though DSA remains a superior follow-up tool for stent-assisted coil embolization. Also, while rare, contrast agents might induce serious adverse events such as anaphylaxis or nephrogenic systemic fibrosis.

There are some limitations to this study. We only examined flow in a stent, though Mizuki et $\mathrm{al}^{17}$ reported that Silent MRA was similar to TOF MRA for assessment of flow outside a stent. $\mathrm{Wu}$ et $\mathrm{al}^{18}$ also reported the feasibility of MRA by using arterial spin-labeling and 3D radial scan data acquisition, which might be somewhat similar to Silent MRA. The average interval between MRA and DSA as a standard of reference is quite long (up to 15 months), which limits the validity of this comparison. Thus, MRA images might not reflect the exact condition of flow in a stent. We were also unable to compare Silent MRA and contrast-enhanced MRA. Finally, we included only cases with a closed-cell-design Enterprise stent in this study. Future studies are required to examine open-cell-design stents because the stent form might produce differences in the MRA images. ${ }^{19}$

These study results may only be preliminary due to the small number of cases examined, limited to the anterior circulation and only 1 stent design. For potential replacement of TOF or contrastenhanced MRA for aneurysm follow-up imaging after treatment, one must analyze not only in-stent flow but also aneurysm occlusion status. We have planned a prospective study to define the situations under which Silent MRA can replace TOF MRA.

\section{CONCLUSIONS}

Silent MRA could visualize flow in an intracranial stent at the anterior circulation more effectively than TOF MRA. Further studies are required to determine the factors that affect MRA images and the most appropriate scan parameters to visualize flow in a stent. Silent MRA might be useful for the follow-up imaging after stent-assisted coil embolization, though some limitations remain to be examined.

\section{REFERENCES}

1. Molyneux A, Kerr R, Stratton I, et al; International Subarachnoid Aneurysm Trial (ISAT) Collaborative Group. International Subarachnoid Aneurysm Trial (ISAT) of neurosurgical clipping versus endovascular coiling in 2143 patients with ruptured intracranial aneurysms: a randomised trial. Lancet 2002;360:1267-74

2. Mine B, Aljishi A, D'Harcour JB, et al. Stent-assisted coiling of unruptured intracranial aneurysms: long-term follow-up in 164 patients with 183 aneurysms. J Neuroradiol 2014;41:322-28

3. Ferns SP, Sprengers ME, van Rooij WJ, et al. Coiling of intracranial aneurysms: a systematic review on initial occlusion and reopening and retreatment rates. Stroke 2009;40:e523-29

4. Kanaan H, Jankowitz B, Aleu A, et al. In-stent thrombosis and ste- nosis after neck-remodeling device-assisted coil embolization of intracranial aneurysms. Neurosurgery 2010;67:1523-32; discussion 1532-33

5. Mocco J, Fargen KM, Albuquerque FC, et al. Delayed thrombosis or stenosis following Enterprise-assisted stent-coiling: is it safe? Midterm results of the interstate collaboration of Enterprise stent coiling. Neurosurgery 2011;69:908-13; discussion 913-14

6. Schaafsma JD, Velthuis BK, Majoie CB, et al. Intracranial aneurysms treated with coil placement: test characteristics of follow-up MR angiography-multicenter study. Radiology 2010;256:209-18

7. Lavoie P, Gariépy JL, Milot G, et al. Residual flow after cerebral aneurysm coil occlusion: diagnostic accuracy of MR angiography. Stroke 2012;43:740-46

8. Cho WS, Kim SS, Lee SJ, et al. The effectiveness of 3T time-of-flight magnetic resonance angiography for follow-up evaluations after the stent-assisted coil embolization of cerebral aneurysms. Acta Radiol 2014;55:604-13

9. Cho YD, Kim KM, Lee WJ, et al. Time-of-flight magnetic resonance angiography for follow-up of coil embolization with Enterprise stent for intracranial aneurysm: usefulness of source images. Korean J Radiol 2014;15:161-68

10. Alibek S, Vogel M, Sun W, et al. Acoustic noise reduction in MRI using Silent Scan: an initial experience. Diagn Interv Radiol 2014; 20:360-63

11. Robson MD, Gatehouse PD, Bydder M, et al. Magnetic resonance: an introduction to ultrashort TE (UTE) imaging. J Comput Assist Tomogr 2003;27:825-46

12. Bergin CJ, Pauly JM, Macovski A. Lung parenchyma: projection reconstruction MR imaging. Radiology 1991;179:777-81

13. Chan CF, Keenan NG, Nielles-Vallespin S, et al. Ultra-short echo time cardiovascular magnetic resonance of atherosclerotic carotid plaque. J Cardiovasc Magn Reson 2010;12:17

14. Gönner F, Heid O, Remonda L, et al. MR angiography with ultrashort echo time in cerebral aneurysms treated with Guglielmi detachable coils. AJNR Am J Neuroradiol 1998;19:1324-28

15. Gönner F, Lövblad KO, Heid O, et al. Magnetic resonance angiography with ultrashort echo times reduces the artefact of aneurysm clips. Neuroradiology 2002;44:755-58

16. Takayama K, Taoka T, Nakagawa H, et al. Usefulness of contrastenhanced magnetic resonance angiography for follow-up of coil embolization with the Enterprise stent for cerebral aneurysms. J Comput Assist Tomogr 2011;35:568-72

17. Mizuki K, Masui T, Katayama M, et al. Evaluation of patients of the cerebral vasculature: comparison with Silenz MRA and 3D TOF MRA. In: Proceedings of Joint Annual Meeting International Society for Magnetic Resonance in Medicine-European Society for Magnetic Resonance in Medicine and Biology, Milan, Italy. May 10-16, 2014

18. Wu H, Block WF, Turski PA, et al. Noncontrast-enhanced threedimensional (3D) intracranial MR angiography using pseudocontinuous arterial spin labeling and accelerated 3D radial acquisition. Magn Reson Med 2013;69:708-15

19. Choi JW, Roh HG, Moon WJ, et al. Optimization of MR parameters of 3D TOF-MRA for various intracranial stents at 3.0T MRI. Neurointervention 2011;6:71-77 